\title{
Binding of Bacillus thuringiensis Cry1 Toxins to the Midgut Brush Border Membrane Vesicles of Chilo suppressalis (Lepidoptera: Pyralidae): Evidence of Shared Binding Sites
}

\author{
LIDIA-MARIANA FIUZA,${ }^{1} \dagger$ CHRISTINA NIELSEN-LEROUX,${ }^{2}$ ERIC GOZÉ, ${ }^{3}$ ROGER FRUTOS,${ }^{1}$ \\ AND JEAN-FRANÇOIS CHARLES ${ }^{2 *}$ \\ BIOTROP-IGEPAM, ${ }^{1}$ and UR-Biométrie et Informatique, ${ }^{3}$ CIRAD, 34032 Montpellier Cedex 1, \\ and Bactéries Entomopathogènes, Institut Pasteur, 75724 Paris Cedex 15, ${ }^{2}$ France
}

Received 5 September 1995/Accepted 8 February 1996

\begin{abstract}
Binding and competition among Cry1Aa, Cry1Ac, and Cry1Ba toxins were analyzed quantitatively in vitro by using ${ }^{125}$ I-labeled activated toxins and brush border membrane vesicles isolated from Chilo suppressalis larval midguts. The three toxins bound specifically to the midgut brush border membrane vesicles. Direct binding experiments showed that Cry1Aa and Cry1Ba recognized a single class of binding sites with different affinities, whereas Cry1Aa recognized two classes of binding sites, one with a high affinity and a low concentration and the other with a lower affinity but higher concentration. Competition experiments showed that toxins Cry1Ac and Cry1Ba shared a binding site in the $C$. suppressalis midgut membranes and that this site was also the low-affinity binding site for Cry1Aa.
\end{abstract}

Bacillus thuringiensis, a soil-dwelling bacterium, synthesizes insecticidal crystal proteins (ICPs), which are also called $\delta$-endotoxins or Cry toxins; these proteins are active against lepidopteran, dipteran, and coleopteran larvae, protozoan pathogens, nematodes, and mites $(5-7,9,12,20)$. After ingestion by insect larvae, the crystalline inclusions first dissolve in the alkaline midgut, and the Cry proteins are proteolytically converted into smaller toxic polypeptides (17). These polypeptides bind to specific binding sites on the apical microvilli of the insect midgut cells before they express their toxicity $(16,32)$. ICP binding sites are involved not only in the specificity of $B$. thuringiensis toxins, but also in at least one type of mechanism of resistance to $B$. thuringiensis $\delta$-endotoxins $(10,33)$. Consequently, there have been numerous studies in which competition between ICPs for binding sites has been investigated ( 8 , $24,32,34)$

The development of transgenic plants that are resistant to insect pests by using $B$. thuringiensis $\delta$-endotoxins as a means of pest control has great potential. Endotoxin genes are expressed in transgenic plants mostly from truncated genes that encode only the toxin moiety of the whole protein, thereby avoiding the problem of solubilization and activation. The best screening test for the activity of toxins produced in plants is therefore the interaction between toxins and binding sites. Specificity, binding site changes, and competition for binding sites are important issues for the development and management of transgenic plants that produce ICPs for crop protection against insect pests (23).

In this study we characterized the activity and specificity of some $B$. thuringiensis $\delta$-endotoxins for the rice striped stem

\footnotetext{
* Corresponding author. Mailing address: Bactéries Entomopathogènes, Institut Pasteur, 25, rue du Dr. Roux, F-75724 Paris Cedex 15, France. Phone: 33-1-40613182. Fax: 33-1-40613044. Electronic mail address: jcharles@pasteur.fr.

$\dagger$ Present address: Centro de Biotechnologia, Universidade Federal do Rio Grande do Sul, Prédio 43421, Campus do Vale/UFRGS, CP 15005, 91501-970 Porto Allegre RS, Brazil.
}

borer, Chilo suppressalis (Lepidoptera: Pyralidae). This insect is a major rice pest in southeast Asia and Japan, as well as in southern Europe, where its causes significant damage in paddy fields in Spain, Italy, and France. Pollution and environmental safety concerns make chemical insecticides undesirable, and $C$. suppressalis is not very susceptible to surface applications of biocontrol agents, such as $B$. thuringiensis, because the insect spends most of its larval stage inside the plant. The transgenic approach is therefore the most promising technique for biological control of the rice striped stem borer. However, recent studies have demonstrated that interactions between toxins and binding sites may vary between target species (8). Thus, development of transgenic rice that is resistant to $C$. suppressalis will require identification of active $B$. thuringiensis toxins, as well as information on the interactions of the toxins with $C$. suppressalis midgut binding sites.

In this paper we describe the toxicity of several B. thuringiensis toxins [Cry1Aa, Cry1Ac, and Cry1Ba, according to the revised nomenclature (3)] for the striped stem borer. In vitro binding assays performed with brush border membrane vesicles (BBMVs) from last-instar larvae in which ${ }^{125}$ I-labeled toxins were used produced evidence that there is competition for a low-affinity binding site among these toxins and that a second binding site is recognized by Cry1Aa.

\section{MATERIALS AND METHODS}

Insects and bioassays. Several thousand larvae of various instars of $C$. suppressalis were collected from rice fields in the Camargue (France) in January 1994 and were used for mass rearing under controlled conditions on an artificial diet (15) in a growth chamber at $25^{\circ} \mathrm{C}$ by using a relative humidity of $70 \%$ and a photoperiod consisting of $16 \mathrm{~h}$ of light and $8 \mathrm{~h}$ of darkness. The population was reared for three generations ( 5 months) in the laboratory before insects were used for bioassays. The bioassays were repeated by using insects from different generations. We collected 10-day-old larvae (second-instar larvae), and averagesize larvae were selected (smaller and larger larvae were rejected), starved for 5 $\mathrm{h}$, and used for bioassays in petri dishes (diameter, $35 \mathrm{~mm}$ ) filled with the artificial diet. Solubilized and activated ICPs (see below) were diluted in sodium phosphate-buffered saline (PBS). Five or six different concentrations plus a control were each tested with 50 larvae (10 larvae per dish). Portions $(100 \mu \mathrm{l})$ of various dilutions of sample (or PBS for controls) were evenly applied onto the diet and allowed to dry. Each test was repeated with three or four different 
batches of larvae from different generations. Levels of mortality were recorded 7 days after treatment. A Probit analysis was performed with SAS/Stat software (29). The Probit model was used to estimate $50 \%$ lethal doses unless a Pearson chi-square test showed a significant lack of fit at $P=0.05$, in which case the normal distribution for susceptibility was replaced by a Gompertz distribution (13). Natural mortality was accounted for by using a maximum-likelihood estimate. The homogeneity of the results obtained with different batches of larvae was checked by performing a likelihood ratio test (18).

Purification and activation of $\boldsymbol{B}$. thuringiensis toxins. Cry1Aa was obtained either from $B$. thuringiensis serovar dendrolimus strain HD37 (17) or from $B$. thuringiensis serovar thuringiensis recombinant strain 407(pHT408) bearing the cry1Aa gene from HD37 (a kind gift from M.-M. Lecadet, Institut Pasteur, Paris, France). B. thuringiensis serovar kurstaki strain HD73 was used to obtain crystals containing the Cry1Ac toxin (1). Cry1Ba was obtained from B. thuringiensis serovar thuringiensis strain 4412 (17).

Bacteria were grown as described by Mahillon and Delcour (21). Autolyzed cultures were centrifuged and washed in $100 \mathrm{mM}$ sodium phosphate buffer ( $\mathrm{pH}$ 7.4) containing $100 \mathrm{mM} \mathrm{NaCl}$ and $0.01 \%$ Triton X-100. Crystals were purified by using 30 to $70 \%$ Renografin gradients. Bands containing pure crystals were collected, extensively washed, resuspended in distilled water containing $0.1 \mathrm{mM}$ phenylmethylsulfonyl fluoride, and stored in aliquots at $-20^{\circ} \mathrm{C}$. Crystal proteins were dissolved by incubating them for $1 \mathrm{~h}$ at $37^{\circ} \mathrm{C}$ in a solution containing $50 \mathrm{mM}$ $\mathrm{Na}_{2} \mathrm{HCO}_{3}, 10 \mathrm{mM}$ dithiothreitol, and $0.1 \mathrm{mM}$ phenylmethylsulfonyl fluoride $(\mathrm{pH}$ 10). The $\mathrm{pH}$ of the solution was then lowered by extensive dialysis against $20 \mathrm{mM}$ Tris ( $\mathrm{pH} 8.6)$, and the crystal proteins were activated by treatment with a bovine pancreatic trypsin (type I; catalog no. T-8003; Sigma) for $2 \mathrm{~h}$ by using $1 \mu \mathrm{g}$ of trypsin per $20 \mu \mathrm{g}$ of protein. The trypsin was then inactivated by adding soybean trypsin inhibitor (type I-S; catalog no. T-9003; Sigma), and the solution was concentrated by ultrafiltration with a Centricon 30 membrane filter (Amicon). The purity of protein samples was checked by performing sodium dodecyl sulfate-10\% polyacrylamide gel electrophoresis (18), and the concentration was determined by the method of Bradford (2) by using bovine serum albumin (BSA) as the standard.

Iodination of $\boldsymbol{B}$. thuringiensis toxins. The toxins were iodinated by the chloramine-T method with Iodo-beads (Pierce) by using the procedure of Markwell (22). A $50-\mu \mathrm{g}$ portion of activated toxin was mixed with $0.5 \mathrm{mCi}$ of $\mathrm{Na}^{125} \mathrm{I}$ (IMS 30; Amersham) in $100 \mu \mathrm{l}$ of carbonate-buffered saline $\left(50 \mathrm{mM} \mathrm{Na}_{2} \mathrm{CO}_{3}, 150 \mathrm{mM}\right.$ $\mathrm{NaCl}, 0.02 \%$ sodium azide; $\mathrm{pH} 10)$ and two Iodo-beads, and the resulting preparation was incubated at room temperature for $5 \mathrm{~min}$ with occasional gentle shaking. The solution was transferred to another tube, and the beads were rinsed twice with $200 \mu \mathrm{l}$ of carbonate-buffered saline $(\mathrm{pH} \mathrm{10)}$. The free iodine was removed by gel filtration on GF-5 columns (Pierce). A sample of each chromatography fraction was subjected to sodium dodecyl sulfate-10 to $15 \%$ polyacrylamide gel electrophoresis (Phast System; Pharmacia), and the gel was autoradiographed at $-80^{\circ} \mathrm{C}$ by using Hyperfilm-MP (Amersham) to evaluate the quality of the labeled toxin preparation. The fractions containing pure ${ }^{125} \mathrm{I}$ labeled toxins were pooled and stored at $4^{\circ} \mathrm{C}$. The specific activities typically were between 80,000 and $110,000 \mathrm{cpm} / \mathrm{pmol}$ of toxin.

Isolation of BBMVs. Fourth-instar and early-fifth-instar C. suppressalis larvae were chilled on ice, and the extremities of the abdomen segments were cut off. The gut was gently pulled out of each body and rinsed in an ice-cold dissection buffer (300 mM mannitol, $5 \mathrm{mM}$ ethyleneglycol-bis- $\beta$-aminoethylether- $N, N$, $N^{\prime}, N^{\prime}$-tetraacetic acid, $20 \mathrm{mM}$ Tris; $\mathrm{pH} 7.4$ ). Freshly isolated midguts were pooled in groups of 50 in $1 \mathrm{ml}$ of dissection buffer and quickly frozen in liquid nitrogen.

The BBMVs were freshly prepared before each series of experiments by using the method of Wolfersberger et al. (35) as modified by Nielsen-LeRoux and Charles (26). The protein contents of the BBMV preparations were determined by the method of Bradford (2) by using BSA as the standard. Each BBMV preparation was checked for leucine aminopeptidase activity (31). The enrichment in leucine aminopeptidase activity between the initial homogenate and the BBMVs was around 6 to $7 \mathrm{U} / \mathrm{U}$ of protein.

Binding experiments. (i) Direct binding experiments. Binding assays were performed under standard conditions, as described by Nielsen-LeRoux and Charles (26). Portions of BBMVs (30 $\mu$ g per polyethylene Microfuge tube) were incubated with the following concentrations of ${ }^{125}$ I-labeled Cry1Aa, Cry1 Ac, and Cry1Ba toxins in Tris-BSA buffer $(20 \mathrm{mM}$ Tris $\mathrm{HCl}, 150 \mathrm{mM} \mathrm{NaCl}, 0.1 \%$ BSA; $\mathrm{pH} 8.5$ ), as follows: 0.1 to $10 \mathrm{nM}$ Cry1Aa, 0.1 to $20 \mathrm{nM}$ Cry1Ac, and 0.5 to $30 \mathrm{nM}$ Cry1Ba. Nonspecific binding was determined in parallel experiments in the presence of a 100:1 mixture of unlabeled and radiolabeled toxins. After incubation for $90 \mathrm{~min}$ at room temperature, the samples were centrifuged at $14,000 \times$ $g$ for $10 \mathrm{~min}$ at $4^{\circ} \mathrm{C}$. The pellets were washed twice in $200 \mu$ l of ice-cold Tris-BSA buffer and then resuspended in $150 \mu \mathrm{l}$ of the same buffer. The experiments were performed in duplicate. Each experimental point was double-counted with a model 1209 liquid scintillation counter (Pharmacia) in microvials containing $3 \mathrm{ml}$ of HiSafe scintillation cocktail (Pharmacia). The binding data were analyzed by using the LIGAND computer program (25) (Biosoft/Elsevier).

(ii) Competition binding experiments. Direct binding experiments were conducted as described previously by using $30 \mu \mathrm{g}$ of BBMVs in a total volume of 100 $\mu \mathrm{l}$ and incubation at room temperature for $90 \mathrm{~min} . \mathrm{IC}_{50} \mathrm{~s}$ (the concentrations of unlabeled toxin that inhibited 50\% specific binding of radiolabeled ICP) were calculated from at least two independent experiments performed in duplicate.
TABLE 1. Toxicities of soluble, activated B. thuringiensis Cry1 proteins to $C$. suppressalis second-instar larvae, as determined by the Gompertz model $^{a}$

\begin{tabular}{ccccccc}
\hline \multirow{2}{*}{$\begin{array}{c}\text { Cry1 } \\
\text { protein }\end{array}$} & $\begin{array}{c}\text { No. of } \\
\text { larvae }\end{array}$ & \multirow{2}{*}{$\begin{array}{c}\mathrm{LC}_{50} \\
\left(\mu \mathrm{g} / \mathrm{cm}^{2}\right)\end{array}$} & $\begin{array}{c}\text { Slope } \\
(\text { mean } \pm \mathrm{SE})\end{array}$ & \multicolumn{3}{c}{ Lack of fit test } \\
\cline { 5 - 8 } & & & & $\chi^{2}$ & $\mathrm{df}$ & $P>\chi^{2}$ \\
\hline Cry1Aa & 1,400 & $1.69(1.35 ; 2.03)^{c}$ & $2.61 \pm 0.19$ & 8.36 & 4 & 0.08 \\
Cry1Ac & 1,200 & $2.24(1.95 ; 2.54)$ & $1.86 \pm 0.12$ & 7.05 & 7 & 0.42 \\
Cry1Ba & $1,050^{d}$ & $2.70(2.23 ; 3.21)$ & $1.48 \pm 0.14$ & 3.35 & 3 & 0.38
\end{tabular}

${ }^{a}$ See text for a description of the Gompertz model.

${ }^{b}$ Total number of larvae analyzed, including controls.

${ }^{c}$ The data in parentheses are the $95 \%$ fiducial limits.

${ }^{d}$ Data for the $0.325-\mu \mathrm{g} / \mathrm{cm}^{2}$ dose were discarded (see text), which reduced the number of larvae analyzed to 900 .

The BBMVs were incubated with fixed concentrations of ${ }^{125}$ I-labeled toxins $(0.2$ $\mathrm{nM}$ Cry1Aa, $0.5 \mathrm{nM}$ Cry1Ac, and $3 \mathrm{nM}$ Cry1Ba) in the presence of a series of concentrations (0.1- to 300 -fold excesses) of unlabeled toxins in Tris-BSA buffer. The value for nonspecific binding (the binding still remaining after the 300 -fold excess of unlabeled toxin was added) was around 10 to $20 \%$ of the total binding value; this value was subtracted from the total binding value to yield the specific binding value. Soluble activated binary toxin from Bacillus sphaericus (26) was used as a negative control.

\section{RESULTS}

Toxicity of $B$. thuringiensis toxins to $C$. suppressalis larvae. The activities of three activated $B$. thuringiensis toxins were tested with second-instar $C$. suppressalis larvae, and 50\% lethal concentrations $\left(\mathrm{LC}_{50}\right)$, fiducial limits, and slopes were estimated (Table 1). Significant discrepancies were found for Cry1Ac when the Probit model was used, so a Gompertz tolerance distribution for levels of susceptibility was used. Neither the normal distribution nor the Gompertz distribution fit all of the Cry1Ba data, because the level of mortality for the lowest dose was too low; this dose was therefore discarded when we estimated the $\mathrm{LC}_{50}$ and the slopes. Both the Probit model and the Gompertz distribution fit the remaining data reasonably well, as well as the Cry1Aa mortality values. To compare $\mathrm{LC}_{50}$ estimated with the same model, the Gompertz distribution was used for all of the toxins. Cry1Aa was significantly more toxic than Cry1Ba, but not significantly more toxic than Cry1Ac; the Cry1Ba and Cry1Ac LC $_{50}$ were not significantly different from each other (Table 1). Homogeneity checks revealed no differences between different generations of larvae.

Direct binding experiments. In direct binding experiments (in which saturation curves were determined), ${ }^{125}$ I-labeled Cry1Aa, Cry1Ac, and Cry1Ba each bound specifically and saturably to BBMVs from $C$. suppressalis midguts. Thus, each of these toxins had at least one specific binding site.

A Scatchard representation of the data (Fig. 1A, inset) suggested that Cry1Aa has two different populations of binding sites, one with a high affinity but a low concentration and the other with a much lower affinity but a higher concentration (Table 2). Only one class of binding site was found for the other two toxins (Fig. 1B and C; Table 2). The binding characteristics of the Cry1Ac site were similar to those of the Cry1Aa high-affinity binding site, whereas the dissociation constant for Cry1Ba was in a range that corresponded to a lower affinity than the affinity for the Cry1Aa and Cry1Ac toxins.

Competition experiments. We also performed homologous competition experiments (between a labeled toxin and its unlabeled analog), as well as heterologous competition experiments (between a labeled toxin and different unlabeled toxins) (Fig. 2). $\mathrm{IC}_{50}$ s were expressed as the molecular ratio of unlabeled competitors to labeled toxins (Table 3).

(i) Homologous competition between ICPs. For toxins 

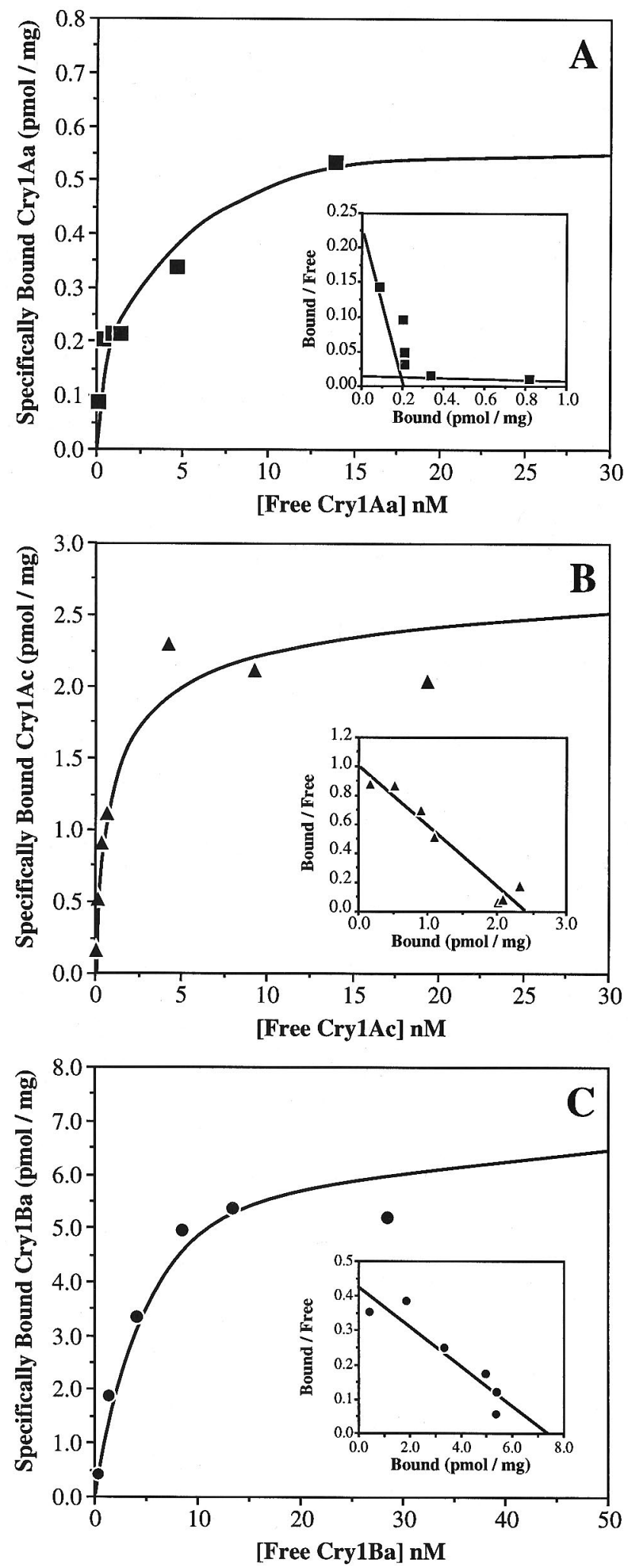

FIG. 1. Results of direct binding experiments performed with ${ }^{125}$ I-labeled $B$. thuringiensis Cry1 toxins and C. suppressalis BBMVs, shown in Scatchard coordinates. Portions $(30 \mu \mathrm{g})$ of membranes were incubated for $90 \mathrm{~min}$ at room temperature with different amounts of ${ }^{125}$ I-labeled Cry1Aa (A), ${ }^{125}$ I-labeled Cry1Ac (B), and ${ }^{125}$ I-labeled Cry1Ba (C). The levels of radioactivity bound to BBMVs were then determined after centrifugation; each value is the mean of the values obtained with duplicate samples. Levels of nonspecific binding were determined with parallel assay mixtures containing excesses of unlabeled toxin Specific binding was calculated by determining the difference between the total binding value (data not shown) and the nonspecific binding value.

Cry1Aa, Cry1Ac, and Cry1Ba, unlabeled homologous toxin was able to displace the binding of ${ }^{125}$ I-labeled toxin (Fig. 2). Under the conditions used, the levels of nonspecific binding were 23, 16, and $19 \%$ for Cry1Aa, Cry1Ac, and Cry1Ba, respectively. The $\mathrm{IC}_{50}$ s were about three times the $K_{d}$ values obtained for each of the labeled toxins, which corresponded to molecular ratios of unlabeled competitor to labeled toxin ranging from 5.5 to 7.6 (Table 3). Thus, the labeled toxins exhibited affinity for the binding sites on the same order of magnitude as the order of magnitude of the corresponding unlabeled toxins. The calculated binding characteristics obtained from these homologous competition experiments (data not shown) were very similar to the characteristics obtained from the direct binding assays and suggested that there are two classes of binding sites for Cry1Aa and only one class of binding site for Cry1Ac and Cry1Ba.

(ii) Heterologous competition for binding sites on $C$. suppressalis. The nonspecific binding values obtained from the homologous competition experiments were used as the baseline values for nonspecific binding in the heterologous competition experiments.

Cry1Ac was able to compete with Cry1Aa (Fig. 2A; Table 3) with a lower efficiency than the homologous toxin. Cry1Ba also competed with Cry1Aa, but at a much lower efficiency.

Labeled Cry1Ac was displaced better by Cry1Ba than by Cry1Aa, but with a lower efficiency than the homologous toxin (Fig. 2B; Table 3). In this case the corresponding $\mathrm{IC}_{50}$ for Cry1Aa was very much higher than the $\mathrm{IC}_{50}$ for unlabeled Cry1Ba.

In contrast, the best competitor for Cry1Ba was Cry1Ac (Fig. 2C; Table 3), which was an even better competitor than the homologous unlabeled toxin. Cry1Aa also inhibited the binding of labeled Cry1Ba, but with a much lower efficiency.

The mosquito larvicidal binary toxin from B. sphaericus (Fig. 2B) did not compete with labeled Cry1Ba.

\section{DISCUSSION}

We investigated the activities of $B$. thuringiensis $\delta$-endotoxins by performing bioassays with second-instar larvae of $C$. suppressalis. The previously reported activities of Cry1Aa and Cry1Ac $(1 \mathrm{a}, 11,19)$ were confirmed. We found that Cry1Ba,

TABLE 2. Binding characteristics of radiolabeled B. thuringiensis ICPs on $C$. suppressalis BBMVs as determined by direct binding experiments ${ }^{a}$

\begin{tabular}{lrc}
\hline $\begin{array}{l}\text { Radiolabeled } \\
\text { ICP or site }\end{array}$ & $K_{d}(\mathrm{nM})$ & $R_{t}(\mathrm{pmol} / \mathrm{mg})$ \\
\hline Cry1Aa site 1 & 0.2 & 0.2 \\
Cry1Aa site 2 & 28.0 & 4.6 \\
Cry1Ac & 0.9 & 2.6 \\
Cry1Ba & 4.9 & 7.6
\end{tabular}

${ }^{a}$ See Fig. 1. 

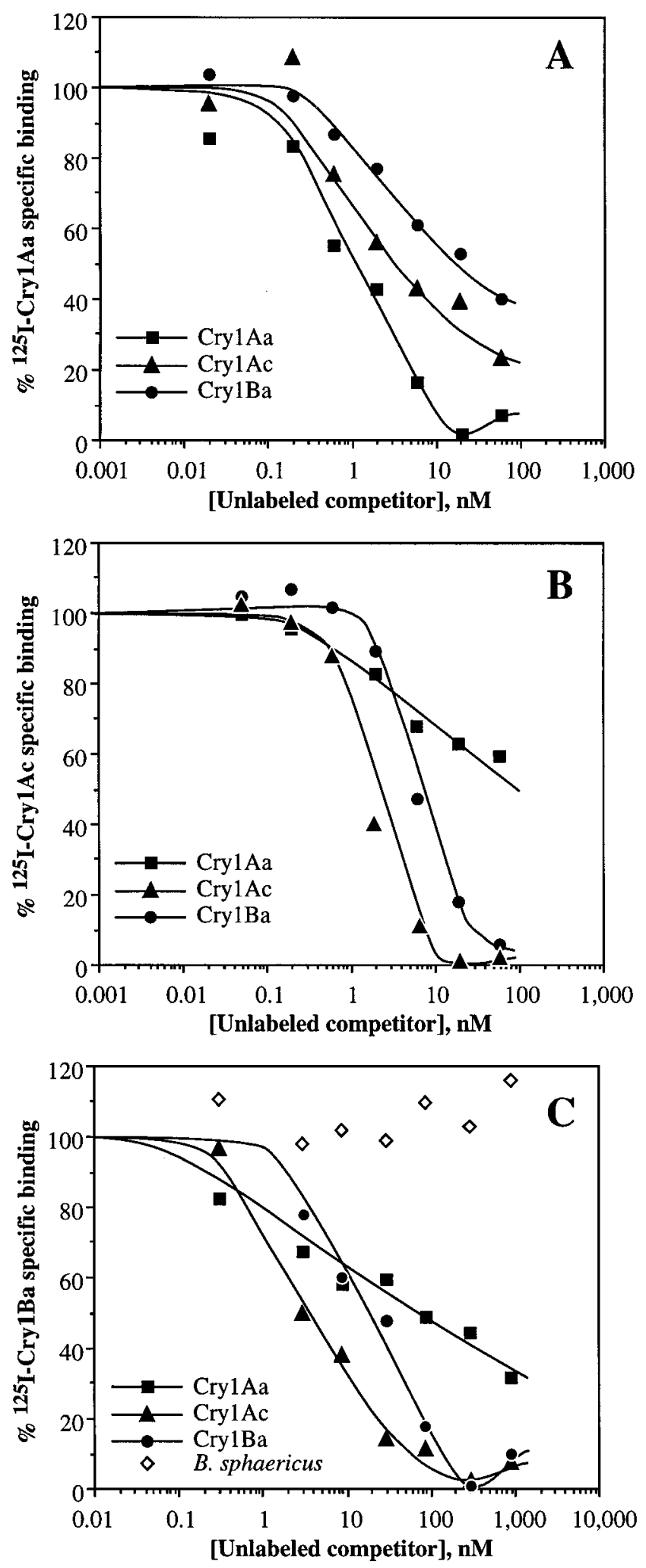

FIG. 2. Competition experiments performed with ${ }^{125}$ I-labeled B. thuringiensis Cry1 toxins and unlabeled B. thuringiensis CryI or B. sphaericus binary toxin competitors. Membranes were incubated for $90 \mathrm{~min}$ at room temperature with a fixed concentration of Cry1Aa (A), Cry1Ac (B), or Cry1Ba (C) that previously had been mixed with unlabeled homologous or heterologous competitor. The levels of radioactivity bound to BBMVs were determined after centrifugation. Each value is the mean of the values obtained with duplicate samples. The displacement curves show the percentages of specific binding of labeled toxin, where $100 \%$ corresponds to the level of binding without competitor and $0 \%$ corresponds to the level of binding following the addition of a 300 -fold excess of unlabeled homologous toxin.

which had not been tested against the striped stem borer previously, was also active.

In vitro investigations of the binding of radiolabeled toxins to stem borer BBMVs have shown that Cry1Aa and Cry1Ac are competitors and thus share a binding site, as suggested by D. H. Dean (3a). We found that Cry1Ba also competes for binding sites with these two toxins. In addition, there is evidence that Cry1Aa recognizes two different binding sites in $C$. suppressalis. One site has a high affinity and is present at a low concentration, and the other site has a low affinity but is present at a high concentration. In heterologous competition experiments performed with Cry1Aa and unlabeled Cry1Ac and Cry $1 \mathrm{Ba}, 20$ and $40 \%$, respectively, of the specific binding remained at the highest competitor concentration used (Fig. 1A). This suggests that there is competition between the toxins only for the Cry1Aa low-affinity binding site. The Cry1Aa lowaffinity binding site may indeed be identical to the binding site for Cry1Ac and Cry1Ba. Thus, the three toxins appear to share the same binding site, but they have different affinities for this site.

Only one binding site was identified for Cry1Ac and Cry1Ba in direct binding assays. Thus, the failure of cold Cry1Aa to displace completely labeled Cry1Ac and Cry1Ba (60 and 30\% of the remaining binding, respectively) (Fig. 2B and C) confirms the lower affinity of Cry1Aa for this putative common low-affinity binding site. The remaining binding of labeled Cry1Ba in the presence of an excess of unlabeled Cry1Aa was higher than the binding of labeled Cry1Ac. This may have been due to the initial difference in toxin concentrations $(0.2 \mathrm{nM}$ labeled Cry1Ac and $3.0 \mathrm{nM}$ labeled Cry1Ba) rather than to a difference in affinity.

There was a good correlation between the $\mathrm{IC}_{50} \mathrm{~s}$ determined in the homologous competition experiments and the affinity values determined in the direct saturation binding assays. The homologous $\mathrm{IC}_{50}$ s for the three toxins were between 5 and 8 (Table 3), which is consistent with the results reported by

TABLE 3. $\mathrm{IC}_{50}$ s from competition experiments between radiolabeled ICP and unlabeled toxins for binding to C. suppressalis $\mathrm{BBMVs}^{a}$

\begin{tabular}{|c|c|c|c|}
\hline \multirow{2}{*}{$\begin{array}{l}\text { Unlabeled } \\
\text { competitor }\end{array}$} & \multicolumn{3}{|c|}{$\mathrm{IC}_{50} \mathrm{~S}$ with the following radiolabeled $\mathrm{ICPs}^{b}$ : } \\
\hline & Cry1Aa & Cry1Ac & Cry1Ba \\
\hline Cry1Aa & 5.5 & 199.2 & 23.6 \\
\hline Cry1Ac & 16.0 & 7.6 & 1.1 \\
\hline Cry1Ba & 74.5 & 27.0 & 6.9 \\
\hline B. sphaericus & $\mathrm{ND}^{c}$ & ND & $\mathrm{NC}^{d}$ \\
\hline
\end{tabular}


Sanchis et al. (28) for the binding of Cry1Ca to BBMVs from Bombyx mori (Bombycidae) and Spodoptera littoralis (Noctuidae).

The presence of common binding sites recognized by the toxins which we studied has implications for questions concerning the complexity of the relationship between affinity and in vivo activity and the variability in binding site responses to similar toxins from one species to another. The affinities of Cry1A toxins vary greatly between target insect midgut binding sites, despite very high levels of amino acid sequence identity. Cry1Ab and Cry1Ac share the same binding site in Trichoplusia $n i$, whereas Cry1Aa specifically binds a different binding site (8). Similarly, Cry1Aa shares a binding site with Cry1Ab and Cry1Ac in Manduca sexta $(16,32)$ and with Cry1Ac in Lymantria dispar (35), but not in Ostrinia nubilalis, in which only $\mathrm{Cry} 1 \mathrm{Ab}$ and Cry1Ac compete for binding sites (4). Cry1Ba, which recognizes the same binding site as Cry1Ac and Cry1Aa in $C$. suppressalis, recognizes a binding site that is different from the Cry1Ac binding site in O. nubilalis (4). These observations suggest that the nature of the binding site or molecule recognized by a particular toxin varies from one species to another and that the relationships between toxins with respect to their binding sites in one species may not be the same in another species. This insect species-related variability has a direct impact on the development of strategies for the use of insect-resistant transgenic plants.

$B$. thuringiensis genes that encode toxins which are active against the striped stem borer (i.e., cry $1 A a$, cry $1 A c$, and $c r y 1 B a$ ) are good candidates for the transformation of rice for insect resistance. In addition to being active against $C$. suppressalis, Cry1Ac is also active against the yellow stem borer, Scirpophaga incertulas, the main rice pest in southeast Asia (19), and therefore affords protection against another species. The main problem to be addressed remains the management of insect resistance when such transgenic plants are used. Several strategies have been proposed. Some workers have proposed that toxin combinations should be used, whereas other workers have recommended that toxins should be rotated $(14,23,27$, $30)$. In the case of $C$. suppressalis, a combination of Cry1Aa plus Cry1Ac or Cry1Aa plus Cry1Ba may be valuable since these toxins compete for one low-affinity binding site. However, there may be a risk associated with the simultaneous use of Cry1Ac and Cry1Ba as these toxins share the same binding site. These arguments are based on the hypothesis that resistance is associated with modifications of the binding site (33), but other mechanisms of resistance are suspected and broadspectrum resistance has already been demonstrated (14). Further investigations of resistance in particular are therefore needed to determine the best strategy.

\section{ACKNOWLEDGMENTS}

We are especially grateful to Marguerite-M. Lecadet (Institut Pasteur) and Nicole Michaux-Ferriere (BIOTROP, CIRAD) for their support and for hosting a large part of this work. We thank Dominique Bordat for providing essential information concerning the rearing of C. suppressalis. We also thank François Leclant, Latifa Drif, Martine Bes, Dolores Triaire, Catherine Fenouillet, and Jacques Escoute for their contributions. We are indebted to Vincent Sanchis (Institut Pasteur) and to Alex Edelman for critical reviews of the manuscript and constructive comments.

This research was supported by the Institut Pasteur, by CIRAD competitive grant ATP 26/93, and by grants from CAPES (Brazil) and from Danish International Development Assistance.

\section{REFERENCES}

1. Adang, M. J., M. J. Staver, T. A. Rocheleau, J. Leighton, R. F. Barker, and D. V. Thomson. 1985. Characterized full-length and truncated plasmid clones of the crystal protein of Bacillus thuringiensis subsp. kurstaki HD-73 and their toxicity to Manduca sexta. Gene 36:289-300.
1a.Aguda, R. (Los Banos, Philippines). Personal communication.

2. Bradford, M. M. 1976. A rapid and sensitive method for the quantitation of microgram quantities of protein utilizing the principle of protein-dye binding. Anal. Biochem. 72:248-254.

3. Crickmore, N., D. R. Zeigler, J. Feitelson, E. Schnepf, B. Lambert, D. Lereclus, C. Gawron-Burke, and D. H. Dean. 1995. Revision of the nomenclature for Bacillus thuringiensis cry genes, p. 14. In Abstracts of the XXVIIIth Annual Meeting of the Society for Invertebrate Pathology. Society for Invertebrate Pathology.

3a.Dean, D. H. (Ohio State University). Personal communication.

4. Denolf, P., S. Jansens, M. Peferoen, D. Degheele, and J. van Rie. 1993. Two different Bacillus thuringiensis delta-endotoxin receptors in the midgut brush border membrane of the European corn borer, Ostrinia nubilalis (Hübner) (Lepidoptera: Pyralidae). Appl. Environ. Microbiol. 59:1828-1837.

5. Dulmage, H. T. 1981. Insecticidal activity of isolates of Bacillus thuringiensis and their potential for pest control, p. 193-222. In H. D. Burges (ed.), Microbial control of pests and plant diseases: 1970-1980. Academic Press, London.

6. Edwards, D., J. Payne, and G. Soares. 1990. U.S. patent 4,948,734.

7. Ellar, D. J., W. E. Thomas, B. H. Knowles, S. Ward, J. Tod, F. A. Drobniewski, J. Lewis, T. Sawyer, D. Last, and C. Nichols. 1985. Biochemistry, genetics, and mode of action of Bacillus thuringiensis $\delta$-endotoxins, p. 230240. In J. Hoch and P. Setlow (ed.), Molecular biology of microbial differentiation. American Society for Microbiology, Washington, D.C.

8. Estada, U., and J. Ferré. 1994. Binding of insecticidal crystal proteins of Bacillus thuringiensis to the midgut brush border of the cabbage looper, Trichoplusia $n i$, and selection for resistance to one of the crystal proteins. Appl. Environ. Microbiol. 60:3840-3846.

9. Feitelson, J. S., J. Payne, and L. Kim. 1992. Bacillus thuringiensis: insects and beyond. Bio/Technology 10:271-276.

10. Ferré, J., M. D. Real, J. Van Rie, S. Jansens, and M. Peferoen. 1991 Resistance to the Bacillus thuringiensis bioinsecticide in a field population of Plutella xylostella is due to a change in a midgut membrane receptor. Proc. Natl. Acad. Sci. USA 88:5119-5123.

11. Fiuza, L. M., N. Michaux-Ferrière, R. Frutos, A. Boets, and J. Van Rie. 1994 Binding of delta-endotoxins from Bacillus thuringiensis to the midgut brush border membrane of the striped stem borer (Chilo suppressalis), p. 398-399. In Abstracts of the XXVIIth Annual Meeting of the Society for Invertebrate Pathology. Society for Invertebrate Pathology.

12. Goldberg, L. J., and J. Margalit. 1977. A bacterial spore demonstrating rapid larvicidal activity against Anopheles sergentii, Uranotaenia unguiculata, Culex univitattus, Aedes aegypti and Culex pipiens. Mosq. News 37:355-358.

13. Gompertz, B. 1825 . On the nature of the function expressive of the law of human mortality. Philos. Trans. R. Soc. Lond. A Math. Phys. Sci. 115:513-580.

14. Gould, F., A. Martinez-Ramirez, A. Anderson, J. Ferré, F. J. Silva, and W. J. Moar. 1992. Broad-spectrum resistance to Bacillus thuringiensis toxins in Heliothis virescens. Proc. Natl. Acad. Sci. USA 89:7986-7990.

15. Guennelon, G., and F. Soria. 1973. Mise au point au laboratoire d'un élevage permanent de la Pyrale du riz Chilo suppressalis Walker (Lepidoptera, Pyralidae) sur milieu artificiel. Ann. Zool. Ecol. Anim. 5:547-558.

16. Hofmann, C., H. Vanderbruggen, H. Höfte, J. Van Rie, S. Jansen, and H. Van Mellaert. 1988. Specificity of Bacillus thuringiensis $\delta$-endotoxins is correlated with the presence of high-affinity binding sites in the brush border membrane of target insect midguts. Proc. Natl. Acad. Sci. USA 85:78447848

17. Höfte, H., and H. R. Whiteley. 1989. Insecticidal crystal proteins of Bacillus thuringiensis. Microbiol. Rev. 53:242-255.

18. Kendall, M. G., and A. Stuart. 1973. Inference and relationship, p. 234-241. In The advanced theory of statistics, 3rd ed., vol. 2. C. Griffin \& Co., London.

19. Lee, M. K., R. Aguda, F. Gould, and D. H. Dean. 1994. Receptor binding properties of Bacillus thuringiensis delta-endotoxins to rice stem borer midguts, p. 36. In Abstracts of the 7th Meeting of the International Program on Rice Biotechnology. Rockefeller Foundation.

20. MacIntosh, S. C., T. B. Stone, S. R. Sims, P. L. Hunst, J. T. Greenplate, P. G. Marrone, F. J. Perlak, D. A. Fischhoff, and R. L. Fuchs. 1990. Specificity and efficacy of purified Bacillus thuringiensis proteins against agronomically important insects. J. Invertebr. Pathol. 56:258-266.

21. Mahillon, J., and J. Delcour. 1984. A convenient procedure for the preparation of highly purified parasporal crystals of Bacillus thuringiensis. J. Microbiol. Methods 3:69-76.

22. Markwell, M. A. K. 1982. A new solid-state reagent to iodinate proteins. I. Conditions for efficient labelling of antiserum. Anal. Biochem. 125:427432.

23. Marrone, P. G., and S. C. MacIntosh. 1993. Resistance to Bacillus thuringiensis and resistance management, p. 221-236. In P. F. Entwisle, J. S. Cory, M. J. Bailey, and S. Higgs (ed.), Bacillus thuringiensis, an environmental biopesticide: theory and practice. John Wiley \& Sons, Chichester, United Kingdom.

24. Martinez-Ramirez, A. C., S. Gonzalez-Nebauer, B. Escribe, and M. D. Real. 1994. Ligand blot identification of a Manduca sexta midgut binding protein specific to three Bacillus thuringiensis CryIA-type ICPs. Biochem. Biophys. Res. Commun. 201:782-787.

25. Munson, P. J., and D. Rodbard. 1980. LIGAND: a versatile computerized 
approach for characterization of ligand-binding systems. Anal. Biochem. 107:220-239.

26. Nielsen-LeRoux, C., and J.-F. Charles. 1992. Binding of Bacillus sphaericus binary toxin to a specific receptor on midgut brush-border membranes from mosquito larvae. Eur. J. Biochem. 210:585-590.

27. Roush, R. T. 1989. Designing resistance management programs: how can you choose? Pestic. Sci. 36:423-441.

28. Sanchis, V., J. Chauffaux, and D. Pauron. 1994. A comparison and analysis of the toxicity and receptor binding properties of Bacillus thuringiensis CryIC delta-endotoxins on Spodoptera littoralis and Bombyx mori. FEBS Lett. 353: 259-263.

29. SAS Institute. 1989. SAS/Stat user's guide, 4th ed., vol. 2, p. 1325-1350. Sas Institute, Cary, N.C

30. Tabashnik, B. E. 1994. Evolution of resistance to Bacillus thuringiensis. Annu. Rev. Entomol. 39:47-79.

31. Tuppy, H., U. Wiesbauer, and E. Wintersberger. 1962. Aminosäure-p-nitroanilide als Substrate für Aminopeptidasen und protelytische Fermente. Hoppe-Seyler's Z. Physiol. Chem. 329:278-288.
32. Van Rie, J., S. Jansen, H. Höfte, D. Degheele, and T. H. Van Mellaert. 1989. Specificity of Bacillus thuringiensis $\delta$-endotoxins. Importance of specific receptors on the brush border membranes of the mid-gut of target insects. Eur. J. Biochem. 186:239-247.

33. Van Rie, J., W. McGaughey, D. Johnson, D. Barnette, and T. H. Van Mellaert. 1989. Mechanism of resistance to the microbial insecticide Bacillus thuringiensis. Science 247:72-74.

34. Wolfersberger, M. G. 1990. Specificity and mode of action of Bacillus thuringiensis insecticidal crystal proteins toxic to lepidopteran larvae: recent insights from studies utilizing midgut brush border membrane vesicles, $\mathrm{p}$. 278-282. In Abstracts of the XXIIIth Annual Meeting of the Society for Invertebrate Pathology. Society for Invertebrate Pathology.

35. Wolfersberger, M. G., P. Lüthy, A. Maurer, P. Parenti, F. V. Sacchi, B. Giordana, and G. M. Hanozet. 1990. The toxicity of two Bacillus thuringiensis $\delta$-endotoxins to gypsy moth larvae is inversely related to the affinity of binding sites on midgut brush border membranes for the toxins. Experimentia 46:301-308. 